\title{
Faster Point Multiplication on Elliptic Curves with Efficient Endomorphisms
}

\author{
Robert P. Gallant ${ }^{1}$, Robert J. Lambert ${ }^{1}$, and Scott A. Vanstone ${ }^{1,2}$ \\ 1 Certicom Research, Canada \\ \{rgallant,rlambert, svanstone\}@certicom.com \\ 2 University of Waterloo, Canada
}

\begin{abstract}
The fundamental operation in elliptic curve cryptographic schemes is the multiplication of an elliptic curve point by an integer. This paper describes a new method for accelerating this operation on classes of elliptic curves that have efficiently-computable endomorphisms. One advantage of the new method is that it is applicable to a larger class of curves than previous such methods. For this special class of curves, a speedup of up to $50 \%$ can be expected over the best general methods for point multiplication.
\end{abstract}

\section{Introduction}

Let $E$ be an elliptic curve defined over a finite field $\mathbb{F}_{q}$. The dominant cost operation in elliptic curve cryptographic schemes is point multiplication, namely computing $k Q$ where $Q$ is an elliptic curve point and $k$ is an integer. This operation is the additive analogue of the exponentiation operation $\alpha^{k}$ in a general (multiplicative-written) finite group. The basic technique for exponentiation is the repeated square-and-multiply algorithm. Numerous methods for speeding up exponentiation and point multiplication have been discussed in the literature; for a survey, see 11/217. These methods can be categorized as follows:

1. Generic methods which can be applied to speed up exponentiation in any finite abelian group, including:

a) Comb techniques (e.g. [15]) which precompute tables which depend on $Q$. Such techniques are applicable when the base point $Q$ is fixed and known a priori, for example in ECDSA signature generation.

b) Addition chains which are useful when $k$ is fixed, for example in RSA decryption.

c) Windowing techniques which are useful when the base point $Q$ is not known a priori, for example in Diffie-Hellman key agreement.

d) Simultaneous multiple exponentiation techniques for computing expressions $k_{1} Q_{1}+k_{2} Q_{2}+\cdots+k_{t} Q_{t}$, for example in ECDSA signature verification.

2. Exponent recoding techniques which replace the binary representation of $k$ with a representation which has fewer non-zero terms (e.g, [10[19]).

3. Methods which are particular to elliptic curve point multiplication such as: 
a) Selection of an underlying finite field which enables faster field arithmetic. For example, selection of a prime field $\mathbb{F}_{p}$ where $p$ is a Mersenne prime or a Mersenne-like prime [31], or an optimal field extension [2].

b) Selection of a representation of the underlying finite field which enables faster field arithmetic. For example, selection of an irreducible trinomial as the reduction polynomial for binary extension fields.

c) Selection of a point representation which enables faster elliptic curve arithmetic [6].

d) Selection of an elliptic curve with special properties, for example Koblitz curves 13 .

Koblitz curves are elliptic curves defined over $\mathbb{F}_{2}$, and were first proposed for cryptographic use in 13. The primary advantage of Koblitz curves is that the Frobenius endomorphism can be exploited to devise fast point multiplication algorithms that do not use any point doublings 30 32. These techniques can be generalized to use arbitrary endomorphisms but are generally not efficient.

The contribution of this paper is a new technique for speeding up point multiplication of elliptic curves having an efficiently-computable endomorphism. While the technique is not as efficient as the methods of Solinas 3032 for Koblitz curves, they are useful for speeding up point multiplication on a larger class of elliptic curves, for example certain curves over prime fields. Such elliptic curves over prime fields have been included in the WAP WTLS (Wireless Transport Layer Security) standard [33. We believe the ideas discussed in this paper are new (though not difficult). In particular, we believe that the approach of decomposing $k$ modulo $n$, and applying just one application of the endomorphism is different than the methods of previous papers. The result is a technique which works on a wider class of curves (in particular, curves defined over prime fields), and works with endomorphisms whose computational cost is not necessarily cheaper than a point operation. For this class of curves, a speedup of up to $50 \%$ can be expected over the best general methods for point multiplication.

The remainder of this paper is organized as follows. $\$ 2$ defines an endomorphism and reviews how the Frobenius endomorphism can be used to speed up point multiplication on Koblitz curves. Our new work for speeding up point multiplication on elliptic curves which have efficiently-computable endomorphisms is described in $\$ 3$ and $\$ 4$. The security of the new method is considered in $\$ 5$ Finally, we draw our conclusions and discuss avenues for future work in 6 .

\section{Endomorphisms}

Let $E$ be an elliptic curve defined over the finite field $\mathbb{F}_{q}$. The point at infinity is

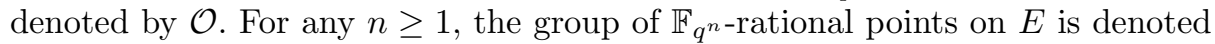
by $E\left(\mathbb{F}_{q^{n}}\right)$.

An endomorphism of $E$ is a rational map $\phi: E \rightarrow E$ satisfying $\phi(\mathcal{O})=\mathcal{O}$ [27]. If the rational map is defined over $\mathbb{F}_{q}$, then the endomorphism $\phi$ is also said to be defined over $\mathbb{F}_{q}$. In this case, $\phi$ is a group homomorphism of $E\left(\mathbb{F}_{q}\right)$, and also of $E\left(\mathbb{F}_{q^{n}}\right)$ for any $n \geq 1$. 
Example 1. Let $E$ be an elliptic curve defined over $\mathbb{F}_{q}$. For each $m \in \mathbb{Z}$ the multiplication by $m$ map $[m]: E \rightarrow E$ defined by $P \mapsto m P$ is an endomorphism defined over $\mathbb{F}_{q}$. A special case is the negation map defined by $P \mapsto-P$.

Example 2. Let $E$ be an elliptic curve defined over $\mathbb{F}_{q}$. Then the $q^{\text {th }}$ power map $\phi: E \rightarrow E$ defined by $(x, y) \mapsto\left(x^{q}, y^{q}\right)$ and $\mathcal{O} \mapsto \mathcal{O}$ is an endomorphism defined over $\mathbb{F}_{q}$, called the Frobenius endomorphism. Since exponentiating to the $q^{\text {th }}$ power is a linear operation in $\mathbb{F}_{q^{n}}$, computation of $\phi(P)$ is normally quite fast. For example, if a normal basis of $\mathbb{F}_{q^{n}}$ over $\mathbb{F}_{q}$ is used, this computation can be implemented as a cyclic shift of the vector representation.

Example $3(\$ 7.2 .3$ of $[5])$. Let $p \equiv 1(\bmod 4)$ be a prime, and consider the elliptic curve

$$
E_{1}: y^{2}=x^{3}+a x
$$

defined over $\mathbb{F}_{p}$. Let $\alpha \in \mathbb{F}_{p}$ be an element of order 4 . Then the map $\phi: E_{1} \rightarrow E_{1}$ defined by $(x, y) \mapsto(-x, \alpha y)$ and $\mathcal{O} \mapsto \mathcal{O}$ is an endomorphism defined over $\mathbb{F}_{p}$. If $P \in E\left(\mathbb{F}_{p}\right)$ is a point of prime order $n$, then $\phi$ acts on $\langle P\rangle$ as a multiplication $\operatorname{map}[\lambda]$, i.e., $\phi(Q)=\lambda Q$ for all $Q \in\langle P\rangle$, where $\lambda$ is an integer satisfying $\lambda^{2} \equiv-1$ $(\bmod n)$. Note that $\phi(Q)$ can be computed using only one multiplication in $\mathbb{F}_{p}$.

Example $4(\{7.2 .3$ of $[5])$. Let $p \equiv 1(\bmod 3)$ be a prime, and consider the elliptic curve

$$
E_{2}: y^{2}=x^{3}+b
$$

defined over $\mathbb{F}_{p}$. Let $\beta \in \mathbb{F}_{p}$ be an element of order 3 . Then the map $\phi: E_{2} \rightarrow E_{2}$ defined by $(x, y) \mapsto(\beta x, y)$ and $\mathcal{O} \mapsto \mathcal{O}$ is an endomorphism defined over $\mathbb{F}_{p}$. If $P \in E\left(\mathbb{F}_{p}\right)$ is a point of prime order $n$, then $\phi$ acts on $\langle P\rangle$ as a multiplication $\operatorname{map}[\lambda]$, where $\lambda$ is an integer satisfying $\lambda^{2}+\lambda \equiv-1(\bmod n)$. Note that $\phi(Q)$ can be computed using only one multiplication in $\mathbb{F}_{p}$.

Example 5 (\$7.2.3 of [5]). Let $p>3$ be a prime such that -7 is a perfect square in $\mathbb{F}_{p}$, and let $\omega=(1+\sqrt{-7}) / 2$, and let $a=(\omega-3) / 4$. Consider the elliptic curve

$$
E_{3}: y^{2}=x^{3}-\frac{3}{4} x^{2}-2 x-1
$$

defined over $\mathbb{F}_{p}$. Then the map $\phi: E_{3} \rightarrow E_{3}$ defined by

$$
(x, y) \mapsto\left(\omega^{-2} \frac{x^{2}-\omega}{x-a}, \omega^{-3} y \frac{x^{2}-2 a x+\omega}{(x-a)^{2}}\right)
$$

and $\mathcal{O} \mapsto \mathcal{O}$ is an endomorphism defined over $\mathbb{F}_{p}$. Computing the endomorphism is a little harder than doubling a point. 
Example 6 ( $\S 14 B$ of $[7])$. Let $p>3$ be a prime such that -2 is a perfect square in $\mathbb{F}_{p}$, and consider the elliptic curve

$$
E_{4}: y^{2}=4 x^{3}-30 x-28
$$

defined over $\mathbb{F}_{p}$. Then the map $\phi: E_{4} \rightarrow E_{4}$ defined by

$$
(x, y) \mapsto\left(-\frac{2 x^{2}+4 x+9}{4(x+2)},-\frac{2 x^{2}+8 x-1}{4 \sqrt{-2}(x+2)^{2}} y\right)
$$

and $\mathcal{O} \mapsto \mathcal{O}$ is an endomorphism defined over $\mathbb{F}_{p}$. Computing the endomorphism is a little harder than doubling a point.

The existing methods $13 \quad 1420 \mid 2932$ for point multiplication which exploit efficiently-computable endomorphisms all use the Frobenius endomorphism. Let $E$ be an elliptic curve defined over a small field $\mathbb{F}_{q}$, and let $\phi$ be the Frobenius endomorphism. To compute $k P$, where $P \in E\left(\mathbb{F}_{q^{n}}\right)$, these methods first compute $k^{\prime}=k \bmod \left(\phi^{n}-1\right)$ in the ring $\mathbb{Z}[\phi]$. Then, one computes a $\phi$-adic expansion $k^{\prime}=\sum_{i=0}^{t} c_{i} \phi^{i}$, where the $c_{i}$ are elements of a small set, e.g., $\{-q / 2, \ldots, q / 2\}$, and $t \approx n$. Finally, $k P$ can be efficiently computed as follows:

$$
k P=k^{\prime} P=\sum_{i=0}^{t} c_{i} \phi^{i}(P) .
$$

The expression (5) can be evaluated using traditional windowing techniques. Observe that the (slow) point doublings in traditional repeated add-and-double algorithms have been replaced by (fast) evaluations of the Frobenius map.

The methods based on Frobenius map expansions can in principle be extended to an arbitrary endomorphism $\psi$. However, these techniques will no longer be efficient if computing $\psi$ is more expensive than a point doubling. Furthermore, one may not have $\psi^{n}-1=0$, so the $\psi$-adic expansion of $k$ may be significantly longer than the binary expansion of $k$. Finally, the existing techniques do not apply when $\operatorname{Norm}(\psi)=1$ (as is the case in Examples 3 and 4 ) since these techniques require a division operation by $\psi$ which yield a nontrivial remainder having norm less than $\operatorname{Norm}(\psi)$.

In the next section, we present a new method that exploits efficientlycomputable endomorphisms such as the ones in Examples 3 , 4, 5and 6 to speed up point multiplication.

\section{Using Efficient Endomorphisms}

Let $E$ be an elliptic curve defined over $\mathbb{F}_{q}$, and let $P \in E\left(\mathbb{F}_{q}\right)$ be a point of prime order $n$. Let $\phi$ be an endomorphism defined over $\mathbb{F}_{q}$, and suppose that the characteristic polynomial of $\phi$ has a root $\lambda$ modulo $n$-since the characteristic polynomial of an endomorphism has degree two we expect that roughly half of all curves will have a root modulo $n$. The map $\phi$ acts on $\langle P\rangle$ as a multiplication $\operatorname{map}[\lambda]$. 
The methods described will be advantageous if computing $\phi$ costs less than computing about $\left(\log _{2} n\right) / 3$ point doublings. In practice, we expect the algorithm to be applied when the cost of $\phi$ is less than (say) 5 point doubles.

The problem we consider is that of computing $k P$ for $k$ selected uniformly at random from the interval $[1, n-1]$. The basic idea of the paper is as follows. Suppose that we can efficiently write $k=k_{1}+k_{2} \lambda \bmod n$, where $k_{1}, k_{2} \in[0,\lceil\sqrt{n}\rceil]$ (see 44). Then we have

$$
\begin{aligned}
k P & =\left(k_{1}+k_{2} \lambda\right) P \\
& =k_{1} P+k_{2}(\lambda P) \\
& =k_{1} P+k_{2} \phi(P) .
\end{aligned}
$$

Now (6) can be computed using any of the 'simultaneous multiple exponentiation' type algorithm 1 , the simplest of which we review below. In the following, $\left(u_{t-1}, \ldots, u_{1}, u_{0}\right)_{2}$ denotes the binary representation of the integer $u$, and $w$ is the window width.

Algorithm 1. Simultaneous multiple point multiplication

INPUT: $w, u=\left(u_{t-1}, \ldots, u_{1}, u_{0}\right)_{2}, v=\left(v_{t-1}, \ldots, v_{1}, v_{0}\right)_{2}, P, Q$.

Output: $u P+v Q$.

1. Compute $i P+j Q$ for all $i, j \in\left[0,2^{w}-1\right]$.

2. Write $u=\left(u^{d-1}, \ldots, u^{1}, u^{0}\right)$ and $v=\left(v^{d-1}, \ldots, v^{1}, v^{0}\right)$ where each $u^{i}$ and $v^{i}$ is a bitstring of length $w$, and $d=\lceil t / w\rceil$.

3. $R \leftarrow \mathcal{O}$.

4. For $i$ from $d-1$ downto 0 do

$4.1 R \leftarrow 2^{w} R$.

$4.2 R \leftarrow R+\left(u^{i} P+v^{i} Q\right)$.

5. $\operatorname{Return}(R)$.

Analysis. Since the bitlengths of $k_{1}$ and $k_{2}$ in (6) are half the bitlength of $k$, we might expect to obtain a significant speedup because we have eliminated a significant number of point doublings at the expense of a few point additions. A precise analysis is complicated due to the large number of point multiplication techniques available. Nevertheless, the following provides some indication of the relative benefits of our method.

Assume that $k$ is a randomly selected $t$-bit integer. When $t=160$, Algorithm 2 of [18] (an exponent recoding and sliding window algorithm) is among the best algorithms for computing $k P$. This method costs approximately 157 point doubles and 34 point additions using windows of size 4 [18. To compare this traditional method with the proposed method, we need an algorithm for computing $k_{1} P+k_{2} Q$ (where in our case $Q=\phi(P)$ ). The following is straightforward and useful for our purposes, but we cannot find a reference for it.

1 These are also known as 'exponentiation using vector-addition chains', 'Shamir's trick', or 'multi-exponentiation'. 
Algorithm 2 of [18] can be combined with the simultaneous multiple exponentiation technique of Algorithm 1 to give an algorithm which is among the best 24 for computing $k_{1} P+k_{2} Q$. Essentially, this combined algorithm computes $\epsilon P$ and $\epsilon Q$ for the integers $\epsilon$ corresponding to allowable windows, then writes each of $k_{1}$ and $k_{2}$ in signed windowed-NAF form as in [18. Finally a left-to-right algorithm is used to iteratively double a common accumulator and add in an $\epsilon P$ or $\epsilon Q$ as appropriate. After $\max \left\{\log _{2} k_{1}, \log _{2} k_{2}\right\}$ iterations, the accumulator holds the desired $k_{1} P+k_{2} Q$.

Using this algorithm in the proposed method to compute $k P=k_{1} P+k_{2}(\lambda P)$ costs approximately 79 point doubles and 38 point additions (when using windows of size 3 [18]) plus 1 evaluation of the map $\phi$. If the cost of a point doubling is 8 field multiplications and the cost of a point addition is 11 field multiplications (as is the case with Jacobian coordinates [4]), then the ratio of the running times of the proposed method to the traditional method is $\approx 0.66$. Thus the new method for point multiplication is roughly $50 \%$ faster than the traditional method when $t=160$. As the bitlength of $k$ increases, the ratio essentially decrease 2 and so the relative performance of the new method gets better. For example, with a bitlength of $t=512$, the ratio is about .62 .

Remark. If computing $\phi$ is cheaper than a point addition, then a few additions can be saved as follows. In the above 'simultaneous windowed-NAF' method for computing $k_{1} P+k_{2} Q$, we initially compute and store points $\epsilon P$ and $\epsilon Q$ for small values of $\epsilon$. If $Q=\phi(P)$, and computing $\phi$ is cheaper than a point addition, then we can instead compute $\epsilon Q=\epsilon \phi(P)=\phi(\epsilon P)$. For example, in the width3 windowed method of [18], computing $k_{1} P+k_{2} \phi(P)$ saves 3 additions at the expense of 3 additional applications of $\phi$.

Example 7. An example of an elliptic curve for which our new method is applicable is

$$
E: y^{2}=x^{3}+3
$$

over the prime field $\mathbb{F}_{p}$, where

$$
p=1461501637330902918203684832716283019655932313743
$$

is a 160 -bit prime, and

$$
\# E\left(\mathbb{F}_{p}\right)=1461501637330902918203687013445034429194588307251
$$

is prime. This curve is included in the WAP specification of the WTLS protocol [33].

${ }^{2}$ There are occasional minor bumps corresponding to window size changes. 


\section{Decomposing $\mathrm{k}$}

In this section we describe an algorithm which takes as input integers $n, \lambda$ and $k \in_{R}[1, n-1]$, and returns integers $k_{1}$ and $k_{2}$ such that $k \equiv k_{1}+k_{2} \lambda(\bmod n)$. The integers $k_{1}$ and $k_{2}$ returned are distinguished in that they are both small or, equivalently, the vector $\left(k_{1}, k_{2}\right) \in \mathbb{Z} \times \mathbb{Z}$ has small Euclidean norm. The term "small" will be made precise below.

Let $G=\mathbb{Z} \times \mathbb{Z}$ and consider the homomorphism $f: G \rightarrow \mathbb{Z}_{n}$ defined by $(i, j) \mapsto(i+\lambda j) \bmod n$. We wish to find a short vector $u \in G$ such that $f(u)=k$; the components of $u$ can then be used as the required $k_{1}$ and $k_{2}$. Note that it is easy to find a vector $v \in G$ such that $f(v)=k ; v=(k, 0)$ is such a vector. The problem is in finding a vector that is also short.

Our approach is the following. We first find linearly independent short vectors $v_{1}, v_{2} \in G$ such that $f\left(v_{1}\right)=f\left(v_{2}\right)=0$. We then find a vector $v$ in the integer lattice generated by $v_{1}$ and $v_{2}$ that is close to $(k, 0)$. It then follows that $u=$ $(k, 0)-v$ is a short vector with $f(u)=f((k, 0))-f(v)=k$. Note that both subproblems can be solved using lattice basis reduction algorithms. However, the direct methods presented here are far less cumbersome to implement.

Finding $\boldsymbol{v}_{\mathbf{1}}$ and $\boldsymbol{v}_{\mathbf{2}}$. The problem of finding two independent short vectors $v_{1}, v_{2}$ such that $f\left(v_{1}\right)=f\left(v_{2}\right)=0$ can be solved using the extended Euclidean algorithm. We apply the extended Euclidean algorithm to find the greatest common divisor of $n$ and $\lambda$. (This gcd is 1 since $n$ is prime.) The algorithm produces a sequence of equations

$$
s_{i} n+t_{i} \lambda=r_{i}, \text { for } i=0,1,2, \ldots,
$$

where $s_{0}=1, t_{0}=0, r_{0}=n, s_{1}=0, t_{1}=1, r_{1}=\lambda$, and $r_{i} \geq 0$ for all $i$. The following properties of the extended Euclidean algorithm are well-known and can be easily proven by induction.

Lemma 1. Let $s_{i}, t_{i}, r_{i}$ be the sequence of variables in (7) produced by an application of the extended Euclidean algorithm to positive integers $n$ and $\lambda$.

(i) $\quad r_{i}>r_{i+1} \geq 0$ for all $i \geq 0$.

(ii) $\left|s_{i}\right|<\left|s_{i+1}\right|$ for $i \geq 1$.

(iii) $\left|t_{i}\right|<\left|t_{i+1}\right|$ for $i \geq 0$.

(iv) $r_{i-1}\left|t_{i}\right|+r_{i}\left|t_{i-1}\right|=n$ for all $i \geq 1$.

Let $m$ be the greatest index for which $r_{m} \geq \sqrt{n}$. Then $r_{m}\left|t_{m+1}\right|+r_{m+1}\left|t_{m}\right|=$ $n$, and $\left|t_{m+1}\right|<\sqrt{n}$. We take $v_{1}=\left(r_{m+1},-t_{m+1}\right)$. By (7) we have $f\left(v_{1}\right)=0$. Also, since $\left|t_{m+1}\right|<\sqrt{n}$ and $\left|r_{m+1}\right|<\sqrt{n}$, we have $\left\|v_{1}\right\| \leq \sqrt{2 n}$. We also take $v_{2}$ to be the shorter of $\left(r_{m},-t_{m}\right)$ and $\left(r_{m+2},-t_{m+2}\right)$. Again by (7), we have $f\left(v_{2}\right)=0$. Heuristically one expects that $v_{2}$ is also short 3 Observe that $v_{1}$ and $v_{2}$ are linearly independent since otherwise if $v_{2}=\left(r_{m},-t_{m}\right)$ (say), then

\footnotetext{
${ }^{3}$ Experiments with various values of $\lambda$ also validate this assumption. It is impossible to prove this without further restrictions; for example consider $\lambda=n-1$.
} 


$$
\frac{r_{m+1}}{r_{m}}=\frac{-t_{m+1}}{-t_{m}}=\frac{t_{m+1}}{t_{m}}
$$

but $r_{m+1} / r_{m}<1$ by Lemma 11(i) and $\left|t_{m+1} / t_{m}\right|>1$ by Lemma 1(iii).

Notice that since $v_{1}$ and $v_{2}$ only depend on $n$ and $\lambda$ (and not on $k$ ), they can be precomputed if $n$ and $\lambda$ are shared domain parameters.

Finding $\boldsymbol{v}$. A vector $v$ in the integer lattice generated by $v_{1}$ and $v_{2}$ that is close to $(k, 0)$ can be easily found using elementary linear algebra[1]. By considering $(k, 0), v_{1}$ and $v_{2}$ as vectors in $\mathbb{Q} \times \mathbb{Q}$, we can write $(k, 0)=\beta_{1} v_{1}+\beta_{2} v_{2}$, where $\beta_{1}, \beta_{2} \in \mathbb{Q}$. Then round $\beta_{1}, \beta_{2}$ to the nearest integers: $b_{1}=\left\lfloor\beta_{1}\right\rceil, b_{2}=\left\lfloor\beta_{2}\right\rceil$. Finally, let $v=b_{1} v_{1}+b_{2} v_{2}$.

The following proves that the vector $u$ is indeed short.

Lemma 2. The vector $u=(k, 0)-v$, where $v$ is constructed as above, has norm at most $\max \left(\left\|v_{1}\right\|,\left\|v_{2}\right\|\right)$.

Proof. We have

$$
\begin{aligned}
u & =(k, 0)-v \\
& =\left(\beta_{1} v_{1}+\beta_{2} v_{2}\right)-\left(b_{1} v_{1}+b_{2} v_{2}\right) \\
& =\left(\beta_{1}-b_{1}\right) v_{1}+\left(\beta_{2}-b_{2}\right) v_{2} .
\end{aligned}
$$

Finally, since $\left|\beta_{1}-b_{1}\right| \leq \frac{1}{2}$ and $\left|\beta_{2}-b_{2}\right| \leq \frac{1}{2}$, by the Triangle Inequality we have

$$
\begin{aligned}
\|u\| & \leq \frac{1}{2}\left\|v_{1}\right\|+\frac{1}{2}\left\|v_{2}\right\| \\
& \leq \max \left(\left\|v_{1}\right\|,\left\|v_{2}\right\|\right) .
\end{aligned}
$$

\section{Security Considerations}

Elliptic curves having efficiently-computable endomorphisms should be regarded as "special" elliptic curves. Using "special" instances of cryptographic schemes is sometimes done for efficiency reasons (for example the use of low encryptionexponent RSA, or the use of small subgroups hidden in a larger group as with DSA). However in any instance of a cryptographic scheme, there is always the chance that an attack will be forthcoming that applies to the special instance and significantly weakens the security. Such is the case here as well.

When selecting an elliptic curve $E$ over $\mathbb{F}_{q}$ for cryptographic use, one must ensure that the order $\# E\left(\mathbb{F}_{q}\right)$ of the elliptic curve is divisible by a large prime number $n$ (say $n \geq 2^{160}$ ) in order to prevent the Pohlig-Hellman [22] and Pollard's rho 2321 attacks. In addition, one must ensure that $\# E\left(\mathbb{F}_{q}\right) \neq q$ in order to prevent the Semaev-Satoh-Araki-Smart attack [26 25 28], and that $n$ does not divide $q^{i}-1$ for all $1 \leq i \leq 20$ in order to prevent the Weil pairing [16] and Tate pairing attacks [8]. Given a curve satisfying these conditions, there is no attack known that significantly reduces the time required to compute elliptic 
curve discrete logarithms. Many such curves having efficient endomorphisms exist and hence appear suitable for cryptographic use. One attack on the elliptic curve discrete logarithm problem on such curves is along the lines of 9 and 34 . The application of such ideas does not reduce the time to compute a logarithm by more than a small factor.

The number of curves for which this technique applies seems to be reasonably large. For instance, one of the Examples 3, 4, 5, and 6 provide a candidate for most primes $p$.

\section{Conclusions and Further Work}

We described a new method for accelerating point multiplication on classes of elliptic curves that have efficiently-computable endomorphisms. The new method for point multiplication is roughly $50 \%$ faster than the best general methods. One advantage of the new method is that it is applicable to a larger class of curves than previous such methods. For example, the method is applicable to classes of curves over prime fields and, in particular, is well suited to two curves over prime fields included in the WAP WTLS specification.

One direction in which our method can be generalized is to use higher powers of the endomorphism. For example, one could write $k \equiv k_{1}+k_{2} \lambda+k_{3} \lambda^{2} \bmod n$ for $t / 3$-bit integers $k_{1}, k_{2}, k_{3}$. This could be done by first finding three linearly independent vectors $v_{1}, v_{2}, v_{3}$ in $\mathbb{Z} \times \mathbb{Z} \times \mathbb{Z}$ each of length roughly $n^{1 / 3}$, and lying in the kernel of the homomorphism $f: \mathbb{Z} \times \mathbb{Z} \times \mathbb{Z}$ defined by $(x, y, z) \mapsto x+y \lambda+z \lambda^{2} \bmod n$. Experimentally, we found that if $\lambda$ satisfies $\lambda^{2}-T \lambda+N \equiv 0 \bmod n$ for a random prime $n$, random $T$ (Trace) and random $N$ (Norm), then the application of LLL to the lattice generated by $\left\{\left(\lambda^{2}, 0,-1\right),(\lambda,-1,0),(0, \lambda,-1),(n, 0,0),(0, n, 0),(0,0, n)\right\}$ results in 3 independent vectors of length about $n^{1 / 3}$, provided at least one of $N, T$ has magnitude at least $n^{1 / 3}$. In this case the application of 'simultaneous multiple exponentiation' type techniques yield an even better improvement over traditional algorithms, with the relevant ratio around $1 / 2$.

We warn that generating $k$ by simply choosing $k_{1}, k_{2}, k_{3}$ first requires care: for example, if $\lambda^{2}+\lambda+1 \equiv 0 \bmod n$ (as in Example 4) then $k_{1}+k_{2} \lambda+k_{3} \lambda^{2} \equiv$ $\left(k_{1}-k_{3}\right)+\left(k_{2}-k_{3}\right) \lambda \bmod n$. Thus simply choosing $k_{1}, k_{2}, k_{3}$ randomly in $\left[0, n^{1 / 3}\right]$ and setting $k \equiv k_{1}+k_{2} \lambda+k_{3} \lambda^{2} \bmod n$ will result in a $k$ having a considerable bias, and consequently the resulting cryptographic scheme may be susceptible to an attack like Bleichenbacher's attack [3] on the DSA as specified in FIPS 186.

Acknowledgements. The authors would like to thank Charles Lam, Alfred Menezes, and John Proos for several very helpful comments and suggestions.

\section{References}

1. L. Babai, "On Lovász' Lattice Reduction and the Nearest Lattice Point Problem", Combinatorica 6 (1986), 1-13 
2. D. Bailey and C. Paar, "Optimal extension fields for fast arithmetic in public-key algorithms", Advances in Cryptology - Crypto '98, 1998, 472-485.

3. D. Bleichenbacher, "On the generation of DSA one-time keys", preprint, November 2000.

4. D. Chudnovsky and G. Chudnovsky, "Sequences of numbers generated by addition in formal groups and new primality and factoring tests", Advances in Applied Mathematics, 7 (1987), 385-434.

5. H. Cohen, A Course in Computational Algebraic Number Theory, Springer-Verlag, 3rd printing, 1996.

6. H. Cohen, A. Miyaji and T. Ono, "Efficient elliptic curve exponentiation using mixed coordinates", Advances in Cryptology-Asiacrypt '98, 1998, 51-65.

7. D. Cox, Primes of the Form $x^{2}+n y^{2}$. Fermat, Class Field Theory and Complex Multiplication, Wiley, 1989.

8. G. Frey and H. Rück, "A remark concerning $m$-divisibility and the discrete logarithm in the divisor class group of curves", Mathematics of Computation, 62 (1994), 865-874.

9. R. Gallant, R. Lambert and S. Vanstone, "Improving the parallelized Pollard lambda search on anomalous binary curves", Mathematics of Computation, 69 (2000), 1699-1705.

10. D. Gollmann, Y. Han and C. Mitchell, "Redundant integer representations and fast exponentiation", Designs, Codes and Cryptography, 7 (1996), 135-151.

11. D. Gordon, "A survey of fast exponentiation methods", Journal of Algorithms, 27 (1998), 129-146.

12. D. Hankerson, J. Hernandez and A. Menezes, "Software implementation of elliptic curve cryptography over binary fields", Proceedings of CHES 2000, LNCS 1965 (2000), 1-24.

13. N. Koblitz, "CM-curves with good cryptographic properties", Advances in Cryptology - Crypto '91, 1992, 279-287.

14. N. Koblitz, "An elliptic curve implementation of the finite field digital signature algorithm", Advances in Cryptology - Crypto '98, 1998, 327-337.

15. C. Lim and P. Lee, "More flexible exponentiation with precomputation", Advances in Cryptology - Crypto '94, 1994, 95-107.

16. A. Menezes, T. Okamoto and S. Vanstone, "Reducing elliptic curve logarithms to logarithms in a finite field", IEEE Transactions on Information Theory, 39 (1993), $1639-1646$.

17. A. Menezes, P. van Oorschot and S. Vanstone, Handbook of Applied Cryptography, CRC Press, 1996.

18. A. Miyaji, T. Ono and H. Cohen, "Efficient elliptic curve exponentiation", Proceedings of ICICS '97, 1997, 282-290.

19. F. Morain and J. Olivos, "Speeding up the computations on an elliptic curve using addition-subtraction chains", Informatique Théorique et Applications, 24 (1990), 531-544.

20. V. Müller, "Fast multiplication in elliptic curves over small fields of characteristic two", Journal of Cryptology, 1 (1998), 219-234.

21. P. van Oorschot and M. Wiener, "Parallel collision search with cryptanalytic applications", Journal of Cryptology, 12 (1999), 1-28.

22. S. Pohlig and M. Hellman, "An improved algorithm for computing logarithms over $G F(p)$ and its cryptographic significance", IEEE Transactions on Information Theory, 24 (1978), 106-110.

23. J. Pollard, "Monte Carlo methods for index computation mod p", Mathematics of Computation, 32 (1978), 918-924. 
24. J. Proos, personal communication, March 2000.

25. T. Satoh and K. Araki, "Fermat quotients and the polynomial time discrete log algorithm for anomalous elliptic curves", Commentarii Mathematici Universitatis Sancti Pauli, 47 (1998), 81-92.

26. I. Semaev, "Evaluation of discrete logarithms in a group of $p$-torsion points of an elliptic curve in characteristic $p "$, Mathematics of Computation, 67 (1998), 353-356.

27. J. Silverman, The Arithmetic of Elliptic Curves, Springer-Verlag, 1986.

28. N. Smart, "The discrete logarithm problem on elliptic curves of trace one", Journal of Cryptology, 12 (1999), 193-196.

29. N. Smart, "Elliptic curve cryptosystems over small fields of odd characteristic", Journal of Cryptology, 12 (1999), 141-151.

30. J. Solinas, "An improved algorithm for arithmetic on a family of elliptic curves", Advances in Cryptology - Crypto '97, 1997, 357-371.

31. J. Solinas, "Generalized Mersenne numbers", Technical Report CORR 99-39, Dept. of C\&O, University of Waterloo, 1999.

32. J. Solinas, "Efficient arithmetic on Koblitz curves", Designs, Codes and Cryptography, 19 (2000), 195-249.

33. WAP WTLS, Wireless Application Protocol Wireless Transport Layer Security Specification, Wireless Application Protocol Forum, February 1999. Drafts available at http://www.wapforum.org

34. M. Wiener and R. Zuccherato, "Faster attacks on elliptic curve cryptosystems", Selected Areas in Cryptography, LNCS 1556 (1999), 190-200. 